\title{
Effectiveness of a lifestyle exercise program for older people receiving a restorative home care service: a pragmatic randomized controlled trial
}

This article was published in the following Dove Press journal:

Clinical Interventions in Aging

29 November 2013

Number of times this article has been viewed

\section{Elissa Burton ${ }^{1,2}$ \\ Gill Lewin ${ }^{1,2}$ \\ Lindy Clemson ${ }^{3}$ \\ Duncan Boldy ${ }^{4}$}

'Faculty of Health Sciences, Curtin University, Perth, WA, Australia;

${ }^{2}$ Research Department, Silver Chain, Perth, WA, Australia; ${ }^{3}$ Health and Work Research Unit, The University of Sydney, Sydney, NSW, Australia; ${ }^{4}$ School of Nursing and Midwifery, Curtin University, Perth, WA, Australia

Correspondence: Elissa Burton Faculty of Health Sciences, Curtin University, GPO Box U1987, Perth, WA 6845, Australia

Tel +6I 892667993

Email e.burton@curtin.edu.au
Background: Restorative home care services are short-term and aimed at maximizing a person's ability to live independently. They are multidimensional and often include an exercise program to improve strength, mobility, and balance. The aim of this study was to determine whether a lifestyle exercise program would be undertaken more often and result in greater functional gains than the current structured exercise program delivered as part of a restorative home care service for older adults.

Methods: A pragmatic randomized controlled trial was conducted in an organization with an established restorative home care service. Individuals who were to have an exercise program as part of their service were randomized to receive either a lifestyle and functional exercise program called LiFE (as this was a new program, the intervention) or the structured exercise program currently being used in the service (control). Exercise data collected by the individuals throughout and pre and post intervention testing was used to measure balance, strength, mobility, falls efficacy, vitality, function, and disability.

Results: There was no difference between the groups in the amounts of exercise undertaken during the 8-week intervention period. Outcome measurement indicated that the LiFE program was as effective, and on $40 \%$ of the measures, more effective, than the structured exercise program.

Conclusion: Organizations delivering restorative home care services that include an exercise component should consider whether LiFE rather than the exercise program they are currently using could help their clients achieve better outcomes.

Keywords: aging, physical activity, reablement, home care services, rehabilitation

\section{Introduction}

As in many countries around the world, Australia's population is aging. It is projected that by 2056, Australians aged 65 years and over will constitute almost a quarter of the population compared with only $13 \%$ in $2007 .{ }^{1}$ As people get older, many will require assistance at some stage to stay living in the community. The Australian Productivity Commission Inquiry report estimated the lifetime risk for an Australian aged 65 years ever requiring an aged care service during their lifetime was $58 \%$. $^{2}$ However, this increased to $80 \%$ for females and $62 \%$ for males aged 85 years. ${ }^{2}$ Currently, over one million older people receive home care services in Australia each year, ${ }^{2}$ and this number is expected to rise in proportion to the anticipated growth in the aging population over the coming decades.

To combat the current and anticipated future increase in the need for home care, restorative home care services were developed. The aim of a restorative home care service is to "create independence, improve self-image and self-esteem, and reduce 
the level of care required" through the delivery of an individualized program. ${ }^{3}$ Restorative home care services are available in the UK, US, Australia, and New Zealand. ${ }^{4}$ The services are generally delivered over 6-12 weeks and comprise a number of components, including a physical activity/ exercise program to assist the older person to regain function and maintain independence. The exercise programs used in restorative home care services are generally "structured" in nature, where structured exercise programs are defined as those which require the client to complete a set number of exercises a number of times each day and a number of times each week.

There are a vast number of structured exercise programs that have been trialed for older community-dwelling people, particularly for the prevention of falls. One well known structured exercise program is the Otago exercise program, which was developed by Campbell et $\mathrm{al}^{5}$ in New Zealand and was designed to prevent falls in community-dwelling older people. Initial research on this home-based strength and balance training program involved 233 women living in the community and aged 80 years and over (Otago exercise group, $n=116$; control group, $n=117) .{ }^{5}$ The program included four visits with a physical therapist and exercises used weight cuffs between $0.5 \mathrm{~kg}$ and $1 \mathrm{~kg}$, and increased in weight as the participants progressed. ${ }^{5}$ Participants completed the exercise three times a week, plus they were asked to undertake a walk outside of their house on another 3 days of the week. The rate of falls significantly decreased for those exercising compared with the control group at both one-year and 2-year follow-up. The Otago exercise program has been shown to be effective in reducing the number of falls and injuries resulting from falls, for both men and women, in four randomized controlled trials (RCTs) involving over 1,000 participants. ${ }^{6}$

Recent research found that many older home care clients prefer to incorporate exercise into their daily routines and tasks, such as housework, gardening, and walking to the shops, rather than completing the more structured, sets, repetitions, and volume-based exercise programs usually delivered in restorative home care services. ${ }^{7}$ Lifestyle or incidental exercise programs have been gaining in popularity over the last few decades and have been shown to result in improved function and a reduction in falls in communitydwelling older people with a history of falling. ${ }^{8-11}$ However, no research has as yet been undertaken to identify whether older people receiving a restorative home care service could benefit more from participating in a lifestyle exercise program compared with a more structured exercise program. Before being able to conduct a study to address this question, it was necessary to conduct a pilot study to ensure that it was feasible to deliver a lifestyle exercise program within a restorative home care service. The pilot found that with some minor changes to the administrative side of the program, the lifestyle and functional exercise program (LiFE) could feasibly be delivered to older people receiving a restorative home care service. ${ }^{12}$ This having been established, the present study was designed to compare the effectiveness of LiFE with the more traditional structured exercise program being used in a restorative home care service. The two hypotheses to be tested were that the lifestyle exercise intervention would be undertaken more often (preferred more) than the structured exercise program and that the lifestyle exercise intervention would result in greater functional gains.

\section{Materials and methods}

A relatively brief methodology is provided here, given that a detailed trial protocol has recently been published. ${ }^{13}$

\section{Study design}

The study was a parallel pragmatic RCT in which the effectiveness of a lifestyle functional exercise program (the intervention) was compared with a structured exercise program (the control) when included in a restorative home care service. Pragmatic trials aim to test an intervention within a "real life" situation and are conducted on people who represent the full spectrum of the population being studied. "If the intervention has a significant effect in a pragmatic trial then it has shown not only that it can work, but that it also works in real life." ${ }^{14}$

\section{Setting and participants}

Silver Chain is an Australian health and community care organization that delivers a number of health care services, including restorative home care services. The restorative home care services are delivered by an interdisciplinary team consisting of occupational therapists, physiotherapists, and registered nurses acting as care managers, and are assisted by aides where required. Silver Chain delivers two restorative home care services to their older clients, ie, the Home Independence Program (HIP) and the Personal Enablement Program (PEP). HIP is delivered to older people living in the community who need short-term assistance to regain their independence, while PEP is delivered to older people who have been in hospital and need a short-term service on discharge to help them return to living independently.

HIP and PEP restorative home care services comprise a number of components, including: chronic disease 
self-management; promotion of active engagement in activities of daily living through work simplification and assistive technology; an exercise program to improve strength, balance, and endurance; falls prevention strategies; improvement and maintenance of skin integrity; and medication, continence, and nutrition management. ${ }^{15} \mathrm{PEP}$ is generally delivered for a maximum of 8 weeks and HIP for 12 weeks. It was expected that the majority of older people who would be involved in this RCT would be PEP clients, referred after hospital discharge because their referral numbers are higher than for HIP.

The study participants were persons living in Perth suburbs (Western Australia) referred for a restorative home care service between August 2011 and April 2012 and who met the RCT inclusion criteria. These criteria were: over 65 years of age; assessed by their care manager as needing an exercise program; not having a diagnosis of dementia or other progressive neurologic disorder; and able to communicate in English.

\section{Sample size and randomization}

The intention was to achieve a total sample size of 150 , with baseline and follow-up data for 75 in each group. The sample size was calculated based on the following assumptions: a $12 \%$ attrition rate (found in the pilot study); ${ }^{12}$ hypothesis tests at the 0.05 level; and an $80 \%$ power to detect "medium" effects $(0.5 \text { standard deviation })^{16}$ in the primary outcome (composite measure, incorporating balance, strength, and mobility). ${ }^{13}$

The randomization process was conducted by a senior researcher not involved in the study. Cases were randomly allocated using the (simple) random number generator in Stata version 10 into the LiFE (intervention) group or the structured exercise (control) group. The randomized cases were then placed in sequentially numbered envelopes.

Recruitment was slower than anticipated, and when it was clear the 150 sample was not going to be achieved even with an extension of the recruitment period by 3 months, and visiting the care managers a number of times to try to understand what was happening and respond to any studyrelated issues, a revision of the sample size was undertaken. It was estimated that 85 was the maximum that was likely to be achieved by the end of the extended recruitment period. It was therefore necessary to rerandomize cases from 50 to 85 to ensure balance among the two groups. Once again the allocation was concealed from the researchers.

\section{Recruitment process and data collection}

When a care manager identified that a client met the study inclusion criteria they gave the client a brief explanation of the study, a letter from the researcher, an information sheet and consent form, and asked the client if they were happy for the researcher to contact them to discuss the project further. If the client agreed, the care manager informed the researcher, who called the client within 3 days to answer questions and set up a time to visit, gain consent, and complete baseline data collection.

Baseline data collection involved the use of eight different outcome measures which are commonly used in studies that examine the effectiveness of exercise programs and have been found to be appropriate for use with older people. ${ }^{17}$ These measures were: functional reach ${ }^{18}$ to measure static balance; chair Sit to Stand 1 and 5 times $^{19}$ for measuring strength; Timed Up and $\mathrm{Go}^{20}$ to assess functional mobility; tandem walk $^{21}$ to measure dynamic balance; Falls Efficacy Scale ${ }^{22}$ for the subject to rate how confident they were that they would not fall when completing daily tasks; the Activities-specific Balance Confidence (ABC) Scale ${ }^{23}$ to ascertain their confidence regarding completing more challenging tasks without falling; the Vitality Plus Scale ${ }^{24}$ to measure any effect on factors such as pain levels, sleep, appetite, and mental and physical well-being; and The Late Life Function ${ }^{25}$ and Disability ${ }^{26}$ instruments to assess individuals' level of function and disability in everyday activities. The scores of interest in these latter instruments were: the function total which is based on the overall functional ability of the participant, ${ }^{25}$ the lower extremity score which is based on tasks such as stair climbing, reaching overhead, standing from a low, soft chair, using a step stool and making a bed, ${ }^{25}$ and the advanced lower extremity score which was based on physical activities that involve a high level of physical ability and endurance, such as walking a mile briskly and walking up multiple levels of stairs. ${ }^{25}$

Once baseline data collection was complete, the researcher opened the envelope which contained the information regarding group allocation and notified the care manager which exercise program should be commenced during their next visit. In addition to the outcomes data collected again at the 8-week follow-up visit, using the same tools as baseline, demographic and service data were extracted from the organization's client database.

Tracking specific client exercises and how many times a day they were completed was trialed during the pilot study. ${ }^{12}$ However, this was found to be too onerous on the client and as such was replaced by a specifically designed calendar. Study participants were asked by their care manager to record how often they did their exercises for the duration of the study. Due to financial constraints, the same researcher 
conducted both the baseline and follow-up home visits and as a consequence was not blinded to group allocation.

\section{Exercise programs}

All care managers undertook a training session outlining their requirements for the study and delivering LiFE to their clients. The training session was led by the researchers and three of the care managers who were part of the pilot study. Care managers had delivered the structured exercise program for a number of years within the restorative home care services and were asked to continue this if the client was randomized to the structured exercise group.

In general, care managers saw their clients a minimum of two times prior to the start of an exercise program and during this time they completed a client functional assessment to determine whether an exercise program was needed. Care managers maintained client progress notes throughout the service; however, these were not specific to the project or used in the study. Care managers offered support and encouragement, not only for the client completing their exercises but for other areas of their restorative home care service, such as removing rugs as tripping hazards and reiterating the importance of clear space, at the next service visit.

\section{LiFE program (intervention)}

The LiFE program was developed to improve balance and increase strength in older community-dwelling people by embedding exercise into everyday activities. ${ }^{8}$ It was also developed as a falls prevention exercise program. ${ }^{27}$ Seven of the activities in the program are designed to challenge balance and six are for improving lower limb strength. The care manager explained the program to the client and described the different exercises it included. How these exercises could be incorporated into their daily routines, and which they would start with, was then discussed and agreed, and the older person was given a manual explaining each of the exercises. Follow-up visits were used to monitor how the client was managing the initial exercises and encouraged to begin doing others. Clients were visited every 10-14 days by their care manager (average three visits), and LiFE was just one aspect of their service that was discussed during these visits. This training and support for implementation was much less than in the original LiFE study. ${ }^{27,28}$

\section{Structured exercise program (control)}

The exercise program being delivered within the restorative home care service at the start of this study was "based" on the Otago falls prevention program developed by Campbell and Robertson, and is called the structured exercise program within this paper. ${ }^{6}$ The program had been modified over time in response to client preferences, to not include weights depending on the client's requirements and sometimes included additional exercises. Table 1 outlines the features of the LiFE program used in this study, the original Otago falls prevention program, and the structured exercise program to show how they differed.

After giving written consent and completing baseline data collection, participants allocated to the current exercise program were given a sheet illustrating (back and front) the exercises (picture), and number of times per day and number of days per week to complete them. The care managers explained the exercises and during follow-up visits reviewed the exercises with the participants.

\section{Data analysis}

Data were analyzed using Statistical Package for the Social Sciences version 19 software (SPSS Inc, Chicago, IL, USA). Both intention-to-treat and per protocol analyses were performed. Intention-to-treat results only are presented except when per protocol results were notably different and then both are presented. Initially paired $t$-tests or Wilcoxon signed-rank tests were employed (depending on normality of data) to determine the functional improvement of clients in their individual exercise group. For each variable, we then measured the change that occurred over the intervention period by subtracting the baseline from the 8-week values. Where the distribution of the change was approximately normal, an independent $t$-test was used to compare the groups. When the data were not normally distributed a Mann-Whitney $U$ test was used. Categorical data were analyzed using a chi-square test.

A new summary variable was created using the functional reach, chair Sit to Stand, Timed Up and Go, and tandem walk variables. ${ }^{13}$ Any change in the summary variable over the 8-week period was assessed for statistical significance in the same manner as the other outcome variables (as described above). Data analysis was supervised by a statistician who was not involved in screening, recruitment, or follow-up of study participants. Statistical significance was determined at $P<0.05$.

\section{Ethics approval}

Ethics approval was obtained from the Curtin University and Silver Chain human research ethics committees prior to commencement of the study. The RCT was registered with the Australian and New Zealand Clinical Trials Registry. ${ }^{29}$

\section{Results}

Figure 1 shows the participant flow for this RCT. A total of 1,993 clients were referred to a restorative home care service 
Table I Features of the exercise programs

\begin{tabular}{|c|c|c|c|}
\hline Features of program & LiFE (for this study) & Otago exercise program & $\begin{array}{l}\text { Structured exercise } \\
\text { program }\end{array}$ \\
\hline Type of exercises & Strength and balance & $\begin{array}{l}\text { Strength and balance, plus walking } \\
\text { for } 30 \text { minutes }\end{array}$ & Strength and balance \\
\hline $\begin{array}{l}\text { Type and frequency } \\
\text { of exercise instructor }\end{array}$ & $\begin{array}{l}\text { Care managers: physiotherapists, } \\
\text { occupational therapists, and nurses. } \\
\text { Average of three visits (not usual practice } \\
\text { for LiFE program) }\end{array}$ & $\begin{array}{l}\text { Physiotherapists and nurses. Four } \\
\text { to five home visits on average }\end{array}$ & $\begin{array}{l}\text { Care managers: } \\
\text { physiotherapists, } \\
\text { occupational therapists, and } \\
\text { nurses. Average three visits }\end{array}$ \\
\hline $\begin{array}{l}\text { Frequency of completing } \\
\text { exercises }\end{array}$ & $\begin{array}{l}\text { Incorporated into daily tasks/routines, } \\
\text { no set amount to be completed; exercise } \\
\text { progressions included, eg, hanging onto } \\
\text { bench top to balance, using one finger } \\
\text { to balance, not holding onto bench top }\end{array}$ & $\begin{array}{l}\text { Begins with one set of } 8-10 \text { repetitions, } \\
\text { increasing to two sets of } \\
8-10 \text { repetitions. The stepping } \\
\text { exercises are one set of } 10 \text { steps } \\
\text { increasing to four sets of } 10 \text { steps }\end{array}$ & $\begin{array}{l}\text { Three times a day, complete } \\
\text { five repetitions. Progress to } \\
\text { level } 2 \text { exercises on back of } \\
\text { sheet, same amount of sets } \\
\text { and repetitions }\end{array}$ \\
\hline $\begin{array}{l}\text { Volume per week and time } \\
\text { per session }\end{array}$ & Every day, no additional time required & $\begin{array}{l}\text { Strength and balance three times } \\
\text { a week, walking twice a week on } \\
\text { different days. Five days in total, } \\
30 \text { minutes each day }\end{array}$ & $\begin{array}{l}\text { Every day, approximately } \\
\text { I5-20 minutes }\end{array}$ \\
\hline Strength exercises & $\begin{array}{l}\text { - Knee bends } \\
\text { - Standing on toes } \\
\text { - Walking on toes } \\
\text { - Standing on heels } \\
\text { - Walking on heels } \\
\text { - Walking up stairs } \\
\text { - Sit to stand } \\
\text { - Sideways walking } \\
\text { - Ankle rotations } \\
\text { - Bend and straightening knees } \\
\text { - Tightening and relaxing buttocks }\end{array}$ & $\begin{array}{l}\text { - Knee strengthening, sit to stand, } \\
\text { knee bends (extensions) } \\
\text { - Back knee strengthening (flexors) } \\
\text { - Side hip strengthening (hip abductors) } \\
\text { - Calf raises (ankle dorsiflexors) } \\
\text { - Toe raises (plantar flexors) } \\
\text { - Stair walking }\end{array}$ & $\begin{array}{l}\text { - Sit to stand } \\
\text { - Side steps } \\
\text { - Stand and reach } \\
\text { - Toe raises } \\
\text { - Backwards walking } \\
\text { - Heel drop }\end{array}$ \\
\hline Balance exercises & $\begin{array}{l}\text { - Tandem stand } \\
\text { - Tandem walking } \\
\text { - One leg stand } \\
\text { - Side to side weight shift } \\
\text { - Forwards and backwards weight shift } \\
\text { - Stepping over objects (including forwards } \\
\text { - Turning backwards and side to side) } \\
\text { - Tunging direction }\end{array}$ & $\begin{array}{l}\text { - Backwards walking } \\
\text { - Walking and turning around } \\
\text { - Sideways walking } \\
\text { - Tandem stance } \\
\text { - Tandem walk } \\
\text { - One leg stand } \\
\text { - Heel walking } \\
\text { - Toe walking } \\
\text { - Heel and toe walking backwards }\end{array}$ & $\begin{array}{l}\text { Toe taps } \\
\text { Salsa knee (lift one leg off } \\
\text { floor) }\end{array}$ \\
\hline $\begin{array}{l}\text { Equipment and instructions } \\
\text { (manual) }\end{array}$ & No equipment, LiFE manual provided & $\begin{array}{l}\text { Ankle cuff weights starting at I kg } \\
\text { increasing to } 8 \mathrm{~kg} \text {, Otago manual } \\
\text { provided }\end{array}$ & $\begin{array}{l}\text { No equipment, a sheet with } \\
\text { exercises illustrated on the } \\
\text { front and back }\end{array}$ \\
\hline Exercise adherence & $\begin{array}{l}\text { Calendar, tick when completed exercises } \\
\text { on that day (not usual practice for LiFE } \\
\text { program) }\end{array}$ & $\begin{array}{l}\text { Calendar, mark date and what exercises } \\
\text { completed, eg, Otago exercises } \\
30 \text { minutes }\end{array}$ & $\begin{array}{l}\text { Calendar, tick when } \\
\text { completed exercises on that } \\
\text { day (not usual practice for } \\
\text { structured program) }\end{array}$ \\
\hline
\end{tabular}

Abbreviation: LiFE, lifestyle and functional exercise.

at Silver Chain between August 2011 and April 2012. One hundred and seven clients were identified as meeting the eligibility criteria, but 27 who met the eligibility criteria declined to participate, due to already being involved in a physiotherapy program $(n=10)$, lack of interest $(n=6)$, no time available $(n=2)$, being stressed $(n=2)$, and a number of other reasons (see Figure 1). Eighty clients randomized to the study were included in the intention-to-treat analysis. Baseline and follow-up data were available for all participants; however, there were two clients at pre-test and five clients at post-test who were unable to complete the physical tests. Four clients were lost to follow-up: one in the LiFE group (family problems) and three in the structured exercise group (no longer interested, health difficulties, and taking too long to receive the program).

\section{Baseline}

The baseline demographics are summarized in Table 2. No significant differences were found between the groups at baseline for any demographic, level of dependency, or outcome measure (see Tables 2 and 3). The average age of the LiFE clients was 80.2 years and that of the structured exercise group was 79.6 years. More women than men were involved 


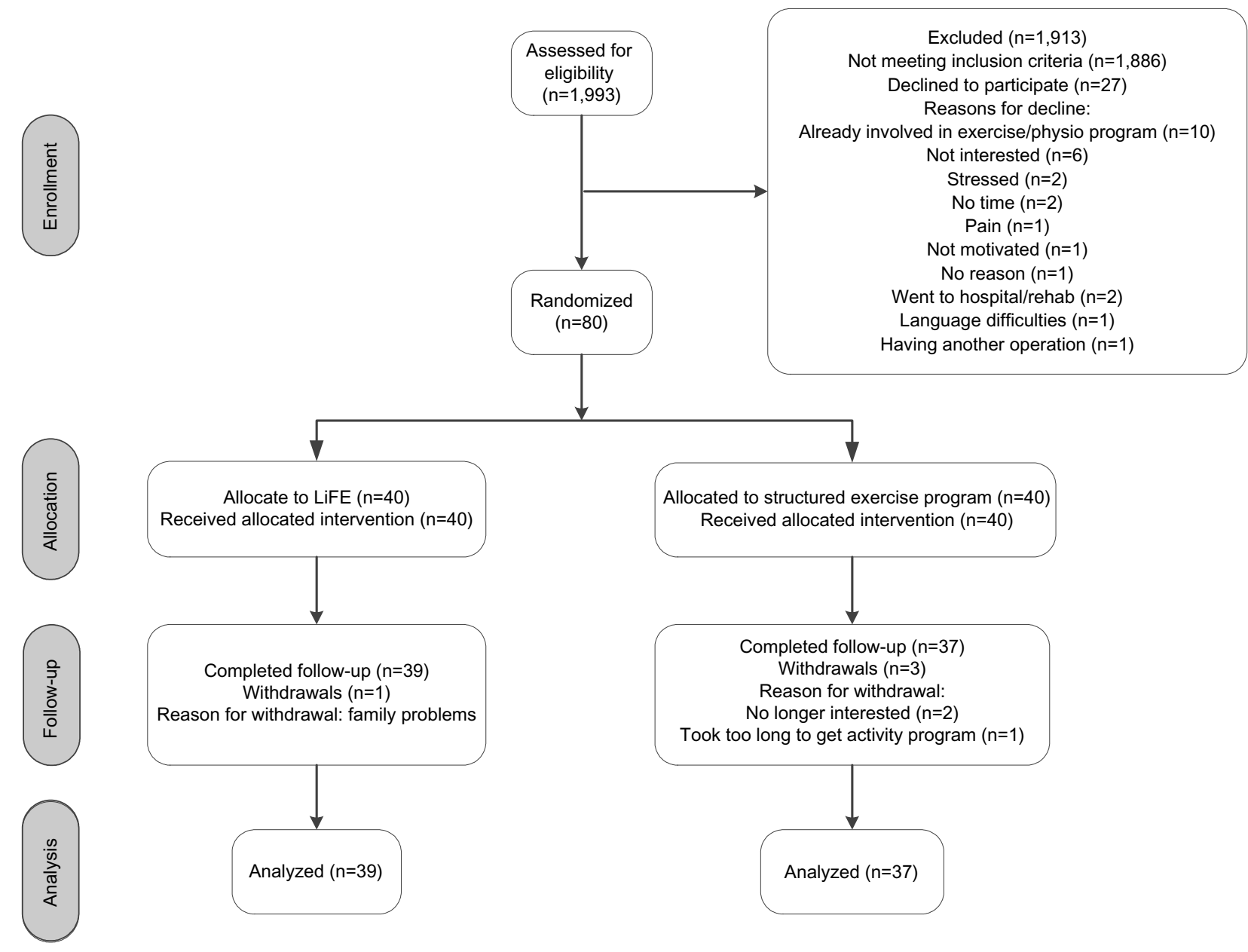

Figure I Participant flow through study.

Abbreviation: LiFE, lifestyle and functional exercise.

in the study for both groups and almost two-thirds of each group (LiFE 60\%; current 67.5\%) lived alone.

Nineteen hundred and ninety-three clients received a restorative home care service during the recruitment period and the demographics and levels of dependency for the population were compared with those of the study sample. No significant differences in demographics (age, sex, language, country of birth, living arrangement, and carer information) were found between clients involved in the RCT and the general restorative home care population for this time. Levels of dependency were also not significantly different.

\section{Exercise program participation}

Three quarters $(n=31)$ of the LiFE clients and two thirds $(n=27)$ of the structured exercise group completed their daily calendars. LiFE clients undertook exercises on average 4.91 times a week during the intervention compared with the structured exercise group who averaged 4.42 times per week. No significant difference was found between the groups in the number of times they exercised during the intervention period.

\section{Outcomes measured}

Table 3 shows that the LiFE group significantly improved in $95 \%$ (19 of 20 ) of the outcome measures during the intervention period, compared with the structured exercise group which significantly improved in 70\% (14 of 20). All of the physical tests showed a significant improvement for clients who participated in the LiFE program, particularly for the summary score, tandem walk, and tandem walk errors $(P<0.001)$, whereas clients in the structured exercise group showed no significant improvement on functional reach and chair Sit to Stand five times or on tandem walk errors.

No difference was seen between the groups at baseline for the summary variable, but the LiFE group was significantly better than the structured exercise group at post-testing $(t[69]=-2.742, P=0.008)$. However, no significant difference was found between the groups for the summary variable when looking at change over time $(t[66]=-1.763, P=0.08)$. When 
Table 2 Demographics

\begin{tabular}{|c|c|c|c|c|c|}
\hline \multirow[t]{2}{*}{ Variables } & \multicolumn{3}{|c|}{ Intention-to-treat $(\mathrm{n}=\mathbf{8 0})$} & \multicolumn{2}{|l|}{ Population } \\
\hline & $\begin{array}{l}\text { LiFE program } \\
\text { (intervention, } n=40 \text { ) }\end{array}$ & $\begin{array}{l}\text { Structured program } \\
\text { (control, } n=40 \text { ) }\end{array}$ & $P$-value & $\begin{array}{l}\text { Restorative care } \\
\text { population }(n=1,993)\end{array}$ & $P$-value \\
\hline Age (years), mean (SD) & $80.2(6.4)$ & $79.58(6.2)$ & 0.659 & $79.05(7.2)$ & 0.254 \\
\hline Sex & & & 0.077 & & $0.28 \mathrm{I}$ \\
\hline Female, n (\%) & $30(75.0)$ & $36(90.0)$ & & $1,530(76.8)$ & \\
\hline Male, n (\%) & $10(25.0)$ & $4(10.0)$ & & $463(23.2)$ & \\
\hline Country of birth & & & 0.145 & & 0.960 \\
\hline Australia, n (\%) & $19(47.5)$ & $27(67.5)$ & & $\mathrm{I}, 089(54.6)$ & \\
\hline England, n (\%) & II (27.5) & $5(12.5)$ & & $359(18.0)$ & \\
\hline Other, n (\%) & $10(25)$ & $8(20)$ & & $545(27.3)$ & \\
\hline Language & & & 0.305 & & 0.273 \\
\hline English-speaking, n (\%) & $37(92.5)$ & $39(97.5)$ & & I,828 (9I.7) & \\
\hline Non-English-speaking, n (\%) & $3(7.5)$ & $\mathrm{I}(2.5)$ & & $116(5.8)$ & \\
\hline Carer availability & & & 0.431 & & 0.874 \\
\hline Has a carer, n (\%) & II (27.5) & $8(20.0)$ & & $450(22.6)$ & \\
\hline Has no carer, n (\%) & $29(72.5)$ & $32(80.0)$ & & $\mathrm{I}, 543(77.4)$ & \\
\hline Living arrangements & & & 0.485 & & 0.447 \\
\hline Lives alone, n (\%) & $24(60.0)$ & $27(67.5)$ & & $\mathrm{I}, 106(55.5)$ & \\
\hline Lives with family/others, n (\%) & $16(40.0)$ & I3 (32.5) & & $813(40.8)$ & \\
\hline Levels of dependency & $(n=37)$ & $(n=37)$ & 0.614 & $(n=1,934)$ & 0.802 \\
\hline Low, n (\%) & $6(16.2)$ & $4(10.8)$ & & $(10.1)$ & \\
\hline Medium, n (\%) & $17(45.9)$ & $21(56.8)$ & & $(55.0)$ & \\
\hline High, n (\%) & $14(37.8)$ & $12(32.4)$ & & (34.9) & \\
\hline
\end{tabular}

Notes: Restorative Home Care Population not stated: language $2.5 \%$; living arrangement $3.7 \%$.

Abbreviations: LiFE, lifestyle and functional exercise; SD, standard deviation.

Table 3 Results of measurement outcomes per exercise group

\begin{tabular}{|c|c|c|c|c|c|c|c|c|}
\hline \multirow{2}{*}{$\frac{\text { Variables }}{\text { Physical activity tests }}$} & \multicolumn{4}{|c|}{ LiFE exercise group } & \multicolumn{4}{|c|}{ Structured exercise group } \\
\hline & Baseline & Post-test & $\begin{array}{l}Z \text { or } \\
t \text { score }\end{array}$ & $P$-value & Baseline & Post-test & $\begin{array}{l}Z \text { or } \\
t \text { score }\end{array}$ & $P$-value \\
\hline Summary score & $-0.10(1.10)$ & $-0.71(0.78)$ & -4.45 & $<0.001 * * *$ & $0.11(0.82)$ & $-0.18(0.93)$ & 2.40 & $0.023^{*}$ \\
\hline Functional reach & $22.74(6.29)$ & $24.74(7.10)$ & -2.22 & $0.032 *$ & $21.29(4.50)$ & $23.01(7.23)$ & 1.72 & 0.095 \\
\hline Sit to Stand I & $3.70(1.70)$ & $3.39(1.99)$ & 2.73 & $0.006 * *$ & $4.29(1.46)$ & $3.80(1.64)$ & 2.63 & $0.012^{*}$ \\
\hline Sit to Stand 5 & $18.45(9.13)$ & $15.68(6.31)$ & 2.51 & $0.012 *$ & $17.60(5.28)$ & $17.61(5.87)$ & -0.46 & 0.644 \\
\hline Timed Up and Go & I3.77 (4.40) & $12.29(3.66)$ & 3.25 & $0.002^{* *}$ & $17.90(7.72)$ & I7.88 (I4.83) & -2.19 & $0.028^{*}$ \\
\hline Tandem walk & $19.62(13.93)$ & $12.92(6.18)$ & -4.34 & $<0.00 I^{* * * *}$ & $18.38(8.10)$ & $16.53(8.98)$ & -1.98 & $0.048^{*}$ \\
\hline Tandem walk errors & $8.47(4.14)$ & $4.42(4.43)$ & 5.31 & $<0.00 I^{* * * *}$ & $9.51(4.12)$ & $8.66(4.14)$ & -1.64 & 0.098 \\
\hline Falls Efficacy Scale & $28.48(14.35)$ & $17.38(9.33)$ & -4.94 & $<0.00 I^{* * * *}$ & $28.35(14.60)$ & $22.40(14.15)$ & -3.02 & $0.002^{* *}$ \\
\hline ABC Scale & $56.37(20.57)$ & $77.52(19.02)$ & 10.07 & $<0.00 I^{* * * *}$ & $52.36(21.08)$ & $65.27(23.27)$ & 4.70 & $<0.00 I^{* * *}$ \\
\hline Vitality Plus Scale & $31.05(7.8 \mathrm{I})$ & $35.42(8.13)$ & -6.15 & $<0.00 I^{* * * *}$ & $29.59(7.37)$ & $31.76(7.02)$ & 2.57 & $0.014^{*}$ \\
\hline \multicolumn{9}{|l|}{ Late Life Disability Instrument } \\
\hline Total disability & $48.94(5.7 I)$ & $50.24(5.07)$ & -2.5 & $0.015^{*}$ & $48.30(7.02)$ & $49.73(6.61)$ & -1.64 & 0.101 \\
\hline Social role & $43.20(8.49)$ & $44.45(8.13)$ & -1.72 & 0.094 & $42.82(10.14)$ & $43.80(9.73)$ & -0.945 & 0.345 \\
\hline Personal role & $55.96(9.39)$ & $60.13(11.78)$ & -2.88 & $0.004 * *$ & $54.55(10.85)$ & $58.94(12.94)$ & -1.82 & 0.069 \\
\hline Limitation & $60.02(9.57)$ & 74.18 (11.53) & -8.42 & $<0.001 * * *$ & $58.09(10.21)$ & $67.36(14.58)$ & -4.24 & $<0.00 I^{* * * *}$ \\
\hline Instrumental role & $58.18(12.50)$ & $74.97(14.00)$ & -5.17 & $<0.001 * * *$ & $54.89(11.29)$ & $65.25(16.03)$ & -4.29 & $<0.00 I^{* * *}$ \\
\hline Management role & $77.69(14.57)$ & $88.88(11.32)$ & -4.69 & $<0.00 I^{* * * *}$ & $80.59(17.5 \mathrm{I})$ & $85.68(15.53)$ & 2.52 & $0.016 *$ \\
\hline \multicolumn{9}{|l|}{ Late Life Function Instrument } \\
\hline Function total & $49.38(6.80)$ & $56.88(8.4 I)$ & -5.23 & $<0.001 * * *$ & $47.09(5.27)$ & $51.13(6.35)$ & -7.40 & $<0.00 I^{* * *}$ \\
\hline Upper extremity & $67.37(15.4 I)$ & 74.07 (I3.38) & -3.46 & $0.001 * *$ & $66.39(13.05)$ & $72.15(12.69)$ & 3.34 & $0.002 * *$ \\
\hline Basic lower extremity & 59.07 (1 I.27) & 71.14 (I3.53) & -8.12 & $<0.00 I^{* * * *}$ & $55.46(9.09)$ & $62.47(12.39)$ & 5.81 & $<0.00 I^{* * *}$ \\
\hline Advanced lower extremity & $30.78(13.43)$ & $42.90(15.39)$ & -5.12 & $<0.00 I^{* * * *}$ & 22.91 (14.87) & $29.74(14.80)$ & 3.25 & $0.002 * *$ \\
\hline
\end{tabular}

Notes: $* P<0.05 ; * * P<0.01 ; * * * P<0.001$.

Abbreviation: LiFE, lifestyle and functional exercise. 
the results of the physical tests were analyzed individually, a significant difference between the groups over time was shown for the tandem walk and the number of errors when completing the tandem walk (Table 4).

Significant differences between the groups were also found on the ABC Scale and the Vitality Plus Scale, which are indicators of improved balance confidence when completing challenging daily activities and of potential healthrelated benefits of exercise, respectively. No difference was found on the Falls Efficacy Scale. The Late Life Function and Disability Instruments measure a number of factors and only one significant difference was found between the groups using the Disability Instrument. The total limitation component, which signifies levels of capability of participating in life tasks, showed that the LiFE group had significantly greater improvement compared with the structured exercise group. There were also significant differences between groups in the instrumental $(Z=-1.98, P=0.048)$ and management $(Z=-2.04, P=0.041)$ role components of the Disability Instrument in the per protocol analysis. These were the only variables in the per protocol analysis showing a significant difference between the groups, where it was not found in the intention-to-treat analysis.
Three functional components of the Late Life Function Instrument (function total, basic lower extremity, and advanced lower extremity) showed the LiFE group improved significantly more than the structured exercise group.

The LiFE group were also found to have a significant reduction in the number of different home care services (for example, domestic assistance, personal care, and meals on wheels) received between baseline and follow-up $(t[74]=-1.99, P=0.049)$ compared with the structured exercise group. This reduction is a crude measure and does not include number of hours received within each service.

\section{Discussion}

Participants in the LiFE program were not found to exercise more frequently than individuals who received a structured exercise program. The first hypothesis we tested did not therefore receive any support. In addition to undertaking similar amounts of exercise, clients in both exercise groups showed significant improvement on many of the functional measures. However, the structured exercise participants only improved on 14 of the 20 measures, whereas the LiFE group improved on 19 measures. These results therefore support our second hypothesis that LiFE would result in greater

Table 4 Results of measurement outcomes over time between the groups

\begin{tabular}{|c|c|c|c|c|c|}
\hline \multirow{2}{*}{$\frac{\text { Variables }}{\text { Physical activity tests }}$} & \multicolumn{5}{|c|}{ Intention-to-treat } \\
\hline & $\begin{array}{l}\text { LiFE } \\
\text { M Diff (SD) }\end{array}$ & $\begin{array}{l}\text { Structured } \\
\text { M Diff (SD) }\end{array}$ & Z or t score & $95 \% \mathrm{Cl}$ & $P$-value \\
\hline Summary score & $-0.55(0.53)$ & $-0.29(0.68)$ & -1.76 & -0.56 to -0.03 & 0.083 \\
\hline Functional reach & $1.99(5.60)$ & $1.73(5.96)$ & 0.199 & $-2.4 \mathrm{I}$ to 2.94 & 0.843 \\
\hline Sit to Stand I & $-0.38(\mathrm{I} .0 \mathrm{I})$ & $-0.49(\mathrm{I} . \mathrm{II})$ & -0.52 & -0.383 to 0.60 & 0.604 \\
\hline Sit to Stand 5 & $-2.05(5.00)$ & $-0.33(4.72)$ & -1.49 & -4.03 to 0.59 & 0.142 \\
\hline Timed Up and Go & $-1.48(2.80)$ & $-0.46(11.54)$ & -0.022 & -4.86 to 2.83 & 0.983 \\
\hline Tandem walk & $-7.07($ ( I I.03) & $-1.33(8.84)$ & -2.15 & -10.47 to -1.00 & $0.032^{*}$ \\
\hline Tandem walk errors & $-4.05(4.70)$ & $-1.12(3.78)$ & -2.90 & -4.95 to -0.91 & $0.005^{* *}$ \\
\hline Falls Efficacy Scale & $-11.49(11.65)$ & $-6.86(13.75)$ & -1.57 & -10.43 to 1.19 & 0.116 \\
\hline ABC Scale & $21.15(13.2)$ & $12.90(16.70)$ & -2.57 & 1.40 to 15.09 & $0.010^{*}$ \\
\hline Vitality Plus Scale & $4.37(4.44)$ & $2.16(5.11)$ & 2.01 & 0.025 to 4.39 & $0.047^{*}$ \\
\hline \multicolumn{6}{|l|}{ Late Life Disability Instrument } \\
\hline Total disability & $1.30(3.20)$ & $1.12(3.59)$ & 0.23 & -1.37 to 1.74 & 0.816 \\
\hline Social role & $1.25(4.54)$ & $0.69(4.44)$ & 0.54 & -1.49 to -2.61 & 0.588 \\
\hline Personal role & $4.11(8.55)$ & $3.80(11.80)$ & -0.53 & -4.38 to 5.01 & 0.595 \\
\hline Limitation & $14.16(10.50)$ & $9.56(10.99)$ & -1.99 & -0.32 to $9.5 \mathrm{I}$ & $0.047^{*}$ \\
\hline Instrumental role & $16.75(13.53)$ & $10.74(12.26)$ & -1.81 & 0.10 to 11.92 & 0.071 \\
\hline Management role & $11.19(14.89)$ & $5.09(12.30)$ & 1.94 & -0.17 to 12.35 & 0.056 \\
\hline \multicolumn{6}{|l|}{ Late Life Function Instrument } \\
\hline Function total & $7.5 \mathrm{I}(5.98)$ & $4.04(3.32)$ & -2.95 & 1.25 to 5.70 & $0.003^{* *}$ \\
\hline Upper extremity & $6.70(12.07)$ & $5.76(10.48)$ & 0.36 & -4.24 to 6.11 & 0.720 \\
\hline Basic lower extremity & $12.07(9.28)$ & $7.01(7.33)$ & 2.63 & 1.22 to 8.90 & $0.010^{*}$ \\
\hline Advanced lower extremity & $12.21(10.85)$ & $6.83(12.77)$ & -2.20 & -0.023 to 10.79 & $0.028^{*}$ \\
\hline
\end{tabular}

Note: $* p<0.05 ; * * p<0.01$.

Abbreviations: $\mathrm{Cl}$, confidence interval; SD, standard deviation; LiFE, lifestyle and functional exercise. 
functional gains than the structured exercise program currently in use in the agency's restorative home care services. The level of improvement on eight of the measures was also larger in the LiFE group. These are discussed in more detail below.

Dynamic balance in the LiFE group improved significantly more than in the structured exercise group. Having poor dynamic balance can contribute to a fear of falling, mobility restrictions, and fall injuries. ${ }^{30}$ Improvements in dynamic balance are important, given that they would be expected to contribute to the older person regaining their independence and improving their confidence, and assist them to remain living in their home.

Improved balance confidence was also found in the LiFE group, because their ABC score was significantly better than in the structured exercise group. The LiFE group's confidence in undertaking more challenging activities, such as riding an escalator, walking through a crowded shopping center, and reaching for items up high within their home was markedly better. Myers et $\mathrm{al}^{31}$ suggest that an $\mathrm{ABC}$ score of $90 \%-100 \%$ is expected for a well older person, $50 \%-80 \%$ indicates a moderate level of physical functioning, and under $50 \%$ suggests a low level of physical functioning. Mean baseline scores for both groups (LiFE 56.37 \pm 20.57 , structured $52.74 \pm 21.72$ ) show they were in the lower end of the moderate level of physical functioning; however, at 8-week post-testing, the LiFE group (77.52 \pm 19.02$)$ was close to the high level of functioning category compared with the structured exercise group (65.22 \pm 23.73$)$. Lajoie and Gallagher ${ }^{32}$ also advise that a score under $67 \%$ may show the older person is at risk of falling or be predictive of a fall in the future. The structured exercise group score was below this threshold, indicating that clients in this group may be at greater risk of a future fall than those in the LiFE group.

The Vitality Plus Scale was developed to measure potential health-related benefits of exercising for older people, that are often missing from other measures, such as improved sleep, higher energy levels, fewer aches and pains, and feeling "good". ${ }^{24}$ Ongoing injury causing pain and feeling too old and tired to exercise have been identified as barriers to older home care clients being active. ${ }^{33}$ The LiFE group displayed significantly greater improvement on this measure compared with the structured exercise group. Because pain and low energy are common experiences for older home care clients, participation in the LiFE program could well benefit all home care clients, not only those receiving a short-term restorative service. Further research is required to determine whether this is the case.
The LiFE group also showed better lower body function than the structured exercise group, and given that both exercise programs were lower body-dominant, this provides further support for our recommendation to the manager of the restorative home care services that the LiFE exercise program should be considered as an option for clients receiving their services.

Our recommendation of LiFE to the restorative home care service manager is also based on the similarity of the study sample to the larger restorative client population shown earlier, indicating that the findings are potentially generalizable to the larger population.

This study can be considered to have two limitations. The first is that the same researcher collected both the baseline and follow-up data and therefore was not blinded to group allocation. However, even if it had been possible to reduce the potential for observer bias through blinding, clients often had their exercise manual or exercise sheet with them and referred to how they were incorporating their exercises into their daily activities.

The second limitation was the smaller than desired sample size, which reduced the study's power to detect change in some of the outcome variables, such as the summary variable, which was trending towards change. The size of the sample was a direct consequence of the lower than expected number of clients being judged appropriate for an exercise program by the care managers during the recruitment period. This had also been an issue during the pilot study, when excessive paperwork associated with the trial and a higher than usual workload at the time of the pilot were identified as the main reasons. ${ }^{12}$ The first issue was addressed by reducing the paperwork needed in the RCT and the second by discussing with the care managers whether for each of them to have eight new clients over the recruitment period on an exercise program would be achievable. They said it would, but this did not turn out to be the case. One of the risks of undertaking a pragmatic RCT in a service that has been operating for a number of years is that organizational structure and service changes may occur during the recruitment and data collection periods, which are beyond the control of the research team. This unfortunately was the case here, with a new service being introduced during the study period, with care managers being asked to act as mentors for the staff of the new service; a turnover of staff (including maternity leave) with new staff taking longer to be trained and recruit clients; and the reduction in workload expected after the pilot study not occurring. A number of strategies were tried to assist the care managers with these issues, but a proportion found their involvement 
in the study too onerous and did not meet their recruitment targets. Although the sample size was smaller than originally calculated, the dropout rate was better than expected $(5 \%$, $\mathrm{n}=4$ compared with $12 \%, \mathrm{n}=15$ expected), and in several instances the sample was still large enough for differences between the exercise programs to emerge.

\section{Conclusion}

Participating in a lifestyle exercise program is at least if not more effective for older restorative home care clients than undertaking a structured exercise program based on sets, repetitions, and volume. The LiFE group performed significantly better on $40 \%$ of the outcome measures. Health practitioners and health and community care organizations that work in services focused on functional improvement should consider the LiFE program for their older clients, particularly for those who suggest they have no extra time available in their day or do not like undertaking structured exercise programs.

\section{Acknowledgments}

EB is supported by a Healthway $\mathrm{PhD}$ research scholarship which includes $\$ 6,500$ (AUD) towards project costs per year. No additional external funding was granted for this study. The authors would like to thank Richard Parsons for his supervision of the data analysis section of the paper, as well as the clients and care managers from Silver Chain who kindly agreed to be involved in the study.

\section{Disclosure}

The authors report no conflicts of interest in this work.

\section{References}

1. Australian Bureau of Statistics. 3222.0 Population projections Australia 2006 to 2101. Available from: http://www.abs.gov.au/Ausstats/abs@. nsf/mf/3222.0. Accessed October 13, 2013.

2. Australian Government Productivity Commission. Caring for Older Australians, Report No 53, Final Inquiry Report. Canberra, Australia: Commonwealth of Australia; 2011. Available from: http://www.pc.gov. au/_data/assets/pdf_file/0004/110929/aged-care-volume1.pdf. Accessed October 13, 2013.

3. Atchison D. Restorative nursing: a concept whose time has come. Nurs Homes. 1992;41:8-12.

4. Lewin G. Restorative home care services. J Curr Clin Care. 2011;3-4: $1-13$.

5. Campbell AJ, Robertson M, Gardner M, Norton R, Tilyard M, Buchner D. Randomised controlled trial of a general practice programme of home based exercise to prevent falls in elderly women. BMJ. 1997;315: $1065-1069$

6. Campbell AJ, Robertson MC. Otago exercise programme to prevent falls in older adults. Wellington: ACC; 2003. Available from: http://www.acc. co.nz/PRD_EXT_CSMP/groups/external_providers/documents/publications_promotion/prd_ctrb118334.pdf. Accessed May 21, 2010.
7. Burton E, Lewin G, Boldy D. Physical activity levels of older people receiving a home care service. J Aging Phys Act. 2013;21:140-154.

8. Clemson L, Fiatarone Singh M, Bundy A, et al. Integration of balance and strength training into daily life activity to reduce rate of falls in older people (the LiFE study): randomised parallel trial. BMJ. 2012;345:e4547.

9. Dunn A, Anderson R, Jakicic J. Lifestyle physical activity interventions. History, short- and long-term effects, and recommendations. Am J Pred Mev. 1998;15:398-412.

10. Opdenacker J, Boen F, De Bourdeaudhuij I, Vanden Auweele Y. Explaining the psychological effects of a sustainable lifestyle physical activity intervention among rural women. Mental Health Phys Act. 2008;1:74-81.

11. Van Roie E, Delecluse C, Opdenacker J, De Bock K, Kennis E, Boen F. Effectiveness of a lifestyle physical activity versus a structured exercise intervention in older adults. J Aging Phys Act. 2010;18:335-352.

12. Burton E, Lewin G, Clemson L, Boldy D. Determining the feasibility of a lifestyle activity program for inclusion in a restorative home care service: a pilot study. Activ Adapt Aging. Epub 2013 June 9.

13. Burton E, Lewin G, Clemson L, Boldy D. Effectiveness of a lifestyle exercise program for older people receiving a restorative home care service: study protocol for a pragmatic randomised controlled trial. BMC Health Serv Res. 2013;13(1):419.

14. Godwin M, Ruhland L, Casson I, et al. Pragmatic controlled clinical trials in primary care: the struggle between external and internal validity. BMC Med Res Methodol. 2003;3:1-7.

15. Silver Chain Nursing Association. Home Independence Program (HIP) User Manual. Perth, Australia: Silver Chain Nursing Association; 2007.

16. Cohen J. Statistical Power Analysis For The Behavioral Sciences. New York, NY: Academic Press; 1969.

17. Johnson C, Myers A, Scholey L, Cyarto EV, Ecclestone N. Outcome evaluation of the Canadian Centre for Activity and Aging's home support exercise program for frail older adults. J Aging Phys Act. 2003;11: 408-424.

18. Duncan P, Weiner D, Chandler J, Studenski S. Functional reach: a new clinical measure of balance. J Gerontol Med Sci. 1990;45: M192-M197.

19. Jette A, Jette D, Ng J, Plotkin D, Bach M; Group TMIMS. Are performance-based measures sufficiently reliable for use in multicenter trials? J Gerontol Med Sci. 1999;54A:M3-M6.

20. Podsiadlo D, Richardson S. The timed up and go: a test of basic functional mobility for frail elderly persons. J Am Geriatr Soc. 1991;39: $142-148$.

21. Guralnik J, Ferrucci L, Pieper C, et al. Lower extremity function and subsequent disability: consistency across studies, predictive models, and value of gait speed alone compared with the short physical performance battery. J Gerontol A Biol Sci Med Sci. 2000;55:M221-M231.

22. Tinetti M, Richman D, Powell L. Falls efficacy as a measure of fear of falling. J Gerontol. 1990;45:239-243.

23. Powell L, Myers A. The Activities-specific Balance Confidence (ABC) Scale. J Gerontol A Biol Sci Med Sci. 1995;50A:M28-M34.

24. Myers A, Malott O, Gray E, et al. Measuring accumulated health-related benefits of exercise participation for older adults: the Vitality Plus Scale. J Gerontol. 1999;54A:M456-M466.

25. Haley S, Jette A, Coster W, et al. Late Life Function and Disability Instrument: II. Development and evaluation of the function component. J Gerontol Med Sci. 2002;57A:M217-M222.

26. Jette A, Haley S, Coster W, et al. Late Life Function and Disability Instrument: I. Development and evaluation of the disability component. J Gerontol Med Sci. 2002;57A(4):M209-M216.

27. Clemson L, Singh M, Bundy A, et al. LiFE pilot study: a randomised trial of balance and strength training embedded in daily life activity to reduce falls in older adults. Aust Occup Ther J. 2010;57:42-50.

28. Clemson L, Singh M, Cumming B, Weissel E, Manollaras K. LiFE: Lifestyle Approach to Reducing Falls Through Exercise. Sydney, Australia: The University of Sydney; 2007. 
29. Burton, E. Physical Activity for Older Home Care Clients Receiving a Restorative Care Program. Available from: https://www.anzctr.org.au/ Trial/Registration/TrialReview.aspx?id=335813\&showOriginal=true Clinical trial identifier: ACTRN12611000788976. Accessed November 4, 2013.

30. Desai A, Goodman V, Kapadia N, Shay B, Szturm T. Relationship between dynamic balance measures and functional performance in community-dwelling elderly people. Phys Ther. 2010;90:748-760.

31. Myers A, Fletcher P, Myers A, Sherk W. Discriminative and evaluative properties of the Activities-specific Balance Confidence (ABC) Scale. J Gerontol Med Sci. 1998;53A:M287-M294.
32. Lajoie Y, Gallagher S. Predicting falls within the elderly community: comparison of postural sway, reaction time, the Berg balance scale and the Activities-specific Balance Confidence (ABC) scale for comparing fallers and non-fallers. Arch Gerontol Geriatr. 2004;38:11-26.

33. Burton E, Lewin G, Boldy D. Barriers and motivators to being physically active for older home care clients. Physical and Occupational Therapy in Geriatrics. 2013;31:21-36.

\section{Publish your work in this journal}

Clinical Interventions in Aging is an international, peer-reviewed journal focusing on evidence-based reports on the value or lack thereof of treatments intended to prevent or delay the onset of maladaptive correlates of aging in human beings. This journal is indexed on PubMed Central, MedLine, the American Chemical Society's 'Chemical Abstracts

\section{Dovepress}

Service' (CAS), Scopus and the Elsevier Bibliographic databases. The manuscript management system is completely online and includes a very quick and fair peer-review system, which is all easy to use. Visit http://www.dovepress.com/testimonials.php to read real quotes from published authors.

Submit your manuscript here: http://www.dovepress.com/clinical-interventions-in-aging-journal 